\title{
Blue rubber bleb nevus syndrome: a single-center case series in 12 years
}

\author{
Haijiao Xia, Jie Wu, Ying Huang \\ Department of Gastroenterology, Children's Hospital of Fudan University, National Children's Medical Center, Shanghai, China \\ Contributions: (I) Conception and design: H Xia, Y Huang; (II) Administrative support: Y Huang; (III) Provision of study materials or patients: H \\ Xia; (IV) Collection and assembly of data: H Xia, J Wu; (V) Data analysis and interpretation: H Xia, J Wu; (VI) Manuscript writing: All author; (VII) \\ Final approval of manuscript: All author. \\ Correspondence to: Ying Huang, MD, PhD. Chief of Gastroenterology, Professor of Pediatrics, Department of Gastroenterology, Children's Hospital \\ of Fudan University, National Children's Medical Center, 399 Wanyuan Road, Shanghai 201102, China. Email: yhuang815@163.com.
}

\begin{abstract}
Background: Blue rubber bleb nevus syndrome is a rare congenital disease characterized by multiple venous malformations in skin and gastrointestinal tract, not all patients have typical cutaneous lesions, refractory anemia may be the only clinical symptom, it is easy to miss diagnosis.

Methods: A retrospective single center study was conducted on 8 patients with blue rubber bleb nevus syndrome from 2009 to 2021. Data were analyzed including clinical feature, diagnostic workup and results, gene detection, treatment and follow-up.

Results: Five children (62.5\%) developed the disease in infancy, which initial symptoms were all cutaneous venous malformations. All children had chronic refractory anemia and gastrointestinal bleeding. Cutaneous lesions were observed in 87.5\%, 1 child had multiorgan involvement. Gastrointestinal venous malformations were observed in $100 \%$, lesions were more common in small intestine than in stomach or colon. No somatic mutation in TEK was found in our children. Diagnostic interval was on average 4.7 years. Eighty-sevenpoint-five percent children received at least one endoscopic or surgical intervention, however, those methods could not eradicate all the lesions and prevent relapse. Two children treated with sirolimus for more than 8 years, only 1 have satisfactory therapeutic effect. Besides, I child has growth retardation and emotional problems during follow-up.

Conclusions: Blue rubber bleb nevus syndrome needs to be considered when find bluish nodular cutaneous lesions, chronic anemia or gastrointestinal bleeding of unknown origin. Capsule endoscopy is the most sensitive in diagnosing of this disease. Oral sirolimus at a relatively low dosage is effective, further comprehensively studies are required to evaluation of its efficacy, safety and the optimal dosage about the children.
\end{abstract}

Keywords: Blue rubber bleb nevus syndrome (BRBNS); venous malformations; anemia; sirolimus; case series

Submitted May 28, 2021. Accepted for publication Aug 26, 2021.

doi: $10.21037 / \mathrm{tp}-21-238$

View this article at: https://dx.doi.org/10.21037/tp-21-238

\section{Introduction}

Blue rubber bleb nevus syndrome (BRBNS) is a rare congenital disease characterized by multiple venous malformations (VMs) in the skin and gastrointestinal (GI) tract or other visceral organs systems, including central nervous system, liver, pancreas, eye, lung, kidney, spleen and thyroid. It was first found by English dermatologist
George Gaskoin in 1818, first reported by Gascoyen in 1860 and 100 years later William Bennett Bean described it in detail and coined the term: a description of the bluish colour of the cutaneous lesions and the rubbery consistency at palpation, also called Bean's syndrome (1). Up to now, around 350 cases of BRBNS with a diverse clinical presentation have been reported world-wide according to 
our review of literatures, we found nearly $21 \%$ of patients with BRBNS were from United States, 11.7\% from China, $8.9 \%$ from Japan, 7.6\% from Spain, 7\% from India, 5.2\% from Turkey and $4.3 \%$ from France, there were also reports from other countries, such as Germany, Portugal, Italy, Canada. It has been identified in all races, with Caucasians most frequently affected.

Multiple blue to bluish-purple soft compressible nodules on skin or mucous membranes which tend to grow in number and size with increasing age, recurrent chronic and intermittent GI bleeding (hematochezia, melena), severe iron deficiency anemia (IDA), are the main symptoms (2), small number of patients may also present with GI complications, such as intussusception, perforation, volvulus with occasional associated mortality (3). In children with BRBNS, because of the lack of specific signs and symptoms, their families often ignore the existence of this disease, only after development of severe melena and anemia, these children will be drawn to seek help from hospitals. Here, we present 8 children with BRBNS aim to describe the main characteristic features, remind clinical doctors of learning more about the clinical manifestations of BRBNS, in order to prevent misdiagnosis and missed diagnosis.

We present the following article in accordance with the AME Case Series reporting checklist (available at https:// dx.doi.org/10.21037/tp-21-238).

\section{Methods}

This was a retrospective study. From March 2009 to March 2021, a total of 8 BRBNS patients were admitted to Children's Hospital of Fudan University. Data on sex, age of presentation, age at diagnosis, current age, clinical and family history, physical and laboratory findings, diagnostic workup and results, genetic testing, treatment, complications and follow-up were collected. Diagnosis of BRBNS was made by the presence of cutaneous and/ or GI vascular malformations and/or by the presence of the characteristic vascular malformations confirmed histologically. Gene detection methods include whole exon sequencing and high throughput sequencing, the specimens were peripheral blood, fresh tissues from operation (ileal VMs), respectively.

Continuous data were presented as the mean and standard deviation or median and interquartile range. Categorical variable data were presented as frequencies and percentages.
The study was conducted in accordance with the Declaration of Helsinki (as revised in 2013). The study was approved by Ethical Committee of Children's Hospital of Fudan University (No.: 2015-130) and informed consent was taken from all patients' parent or legal guardian.

\section{Results}

Table 1 summarizes the main characteristics, management and clinical outcome of the patients.

\section{Patients group}

In total of 8 patients, the average presentation age was 2.6 years, the mean diagnosis age was 7.3 years, diagnostic interval (presentation to diagnosis) was on average 4.7 years (range, 0.5 to 13.5 years). There was no family history identified in our group.

\section{Clinical presentation}

Cutaneous lesions were present in $7 / 8$ patients $(87.5 \%)$, it appeared as the initial symptom in 6 patients (Figure 1), 1 patient (\#2) skin lesions occurred in the 7th year of the course of disease. Skin lesions were scattered, extremities, trunk and head were most frequently involved. Most patients were not typical, mainly blue vascular papular lesions measuring several millimeters in size, and with few number (only 1-5 skin lesions), which would be ignored or regarded as ordinary pigmented mole. Only 1 patient (\#1) had several round rubbery lesions, initially the size of the lesion was about the size of a sesame and gradually increase in size and causing pain over the years, the biggest lesion was over the left elbow. All patients (100\%) presented with chronic recurring anemia manifesting as pallor, fatigue or poor exercise tolerance. GI bleeding occurred in all patients (100\%).

Other organs involvement included hepatic in $3(37.5 \%)$, intra-muscular in $2(25.0 \%)$, ocular, thyroid, splenic, intraosseous, pulmonary in $1(12.5 \%)$ respectively. Nontherapy related complication was intussusception occur in 1 patient (\#1, 3 episodes).

\section{Laboratory investigations}

Complete blood counts examinations revealed severe anemia in all patients, range of whose hemoglobin $(\mathrm{Hb})$ values fluctuation were shown in Table 1. Serum iron 


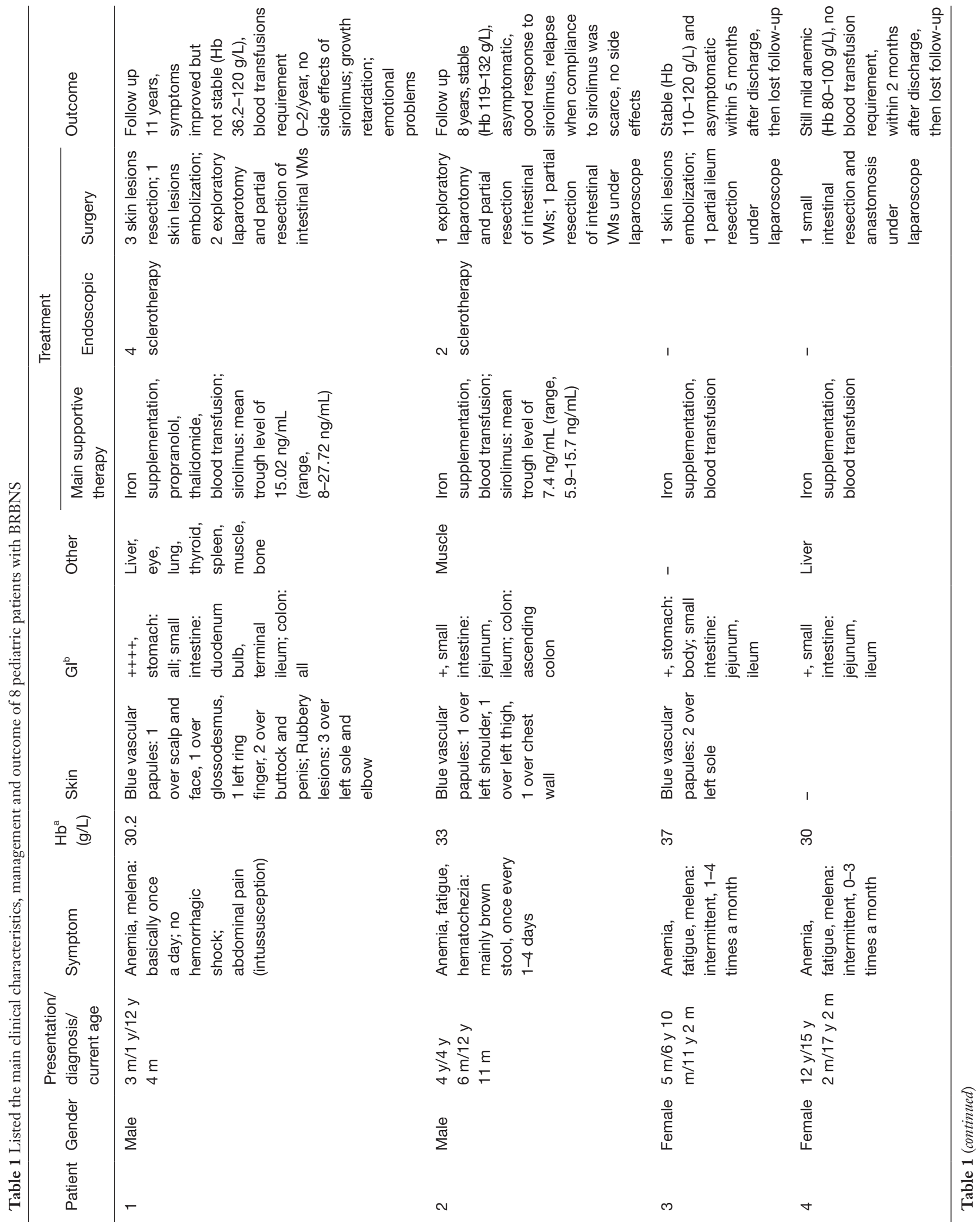




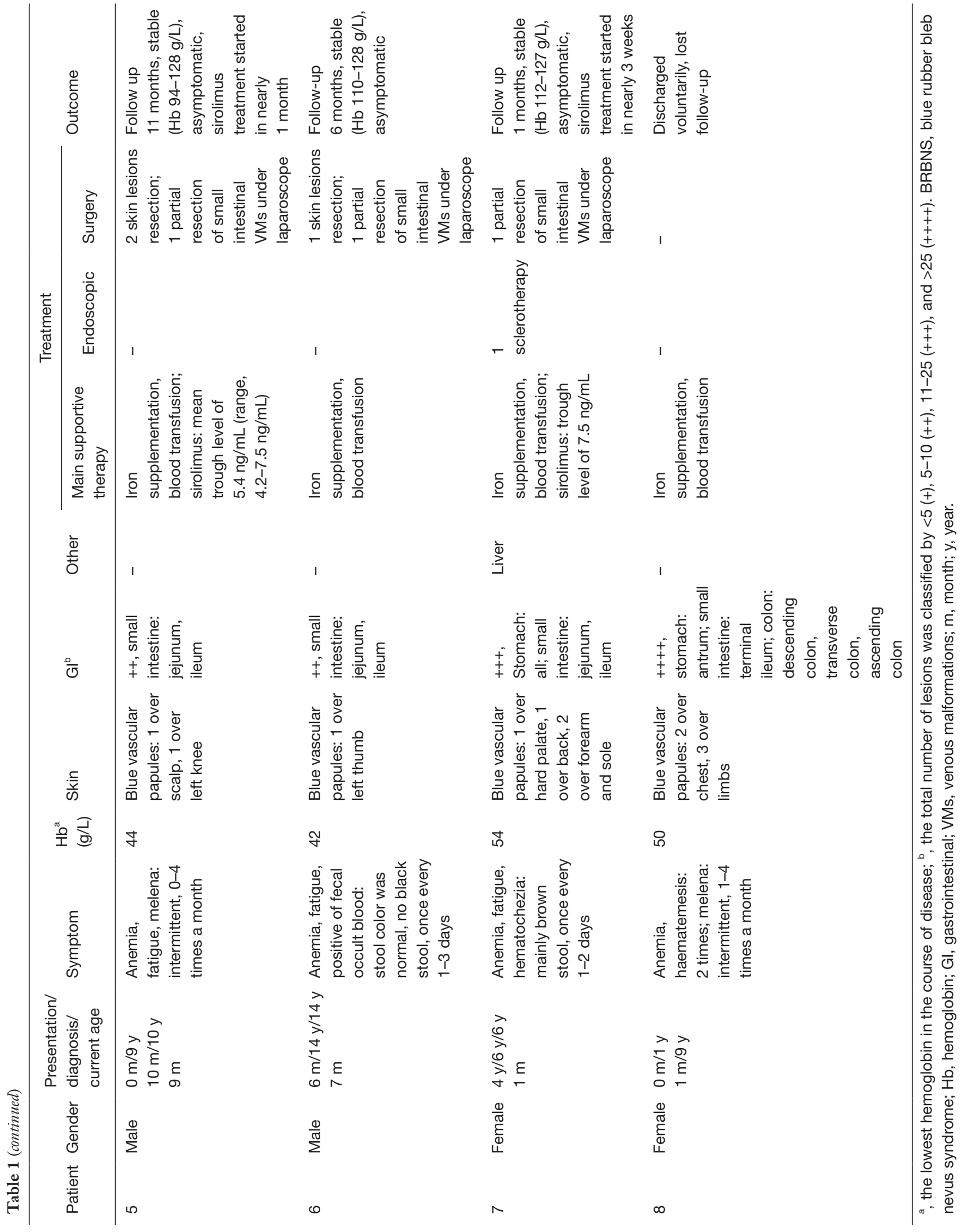



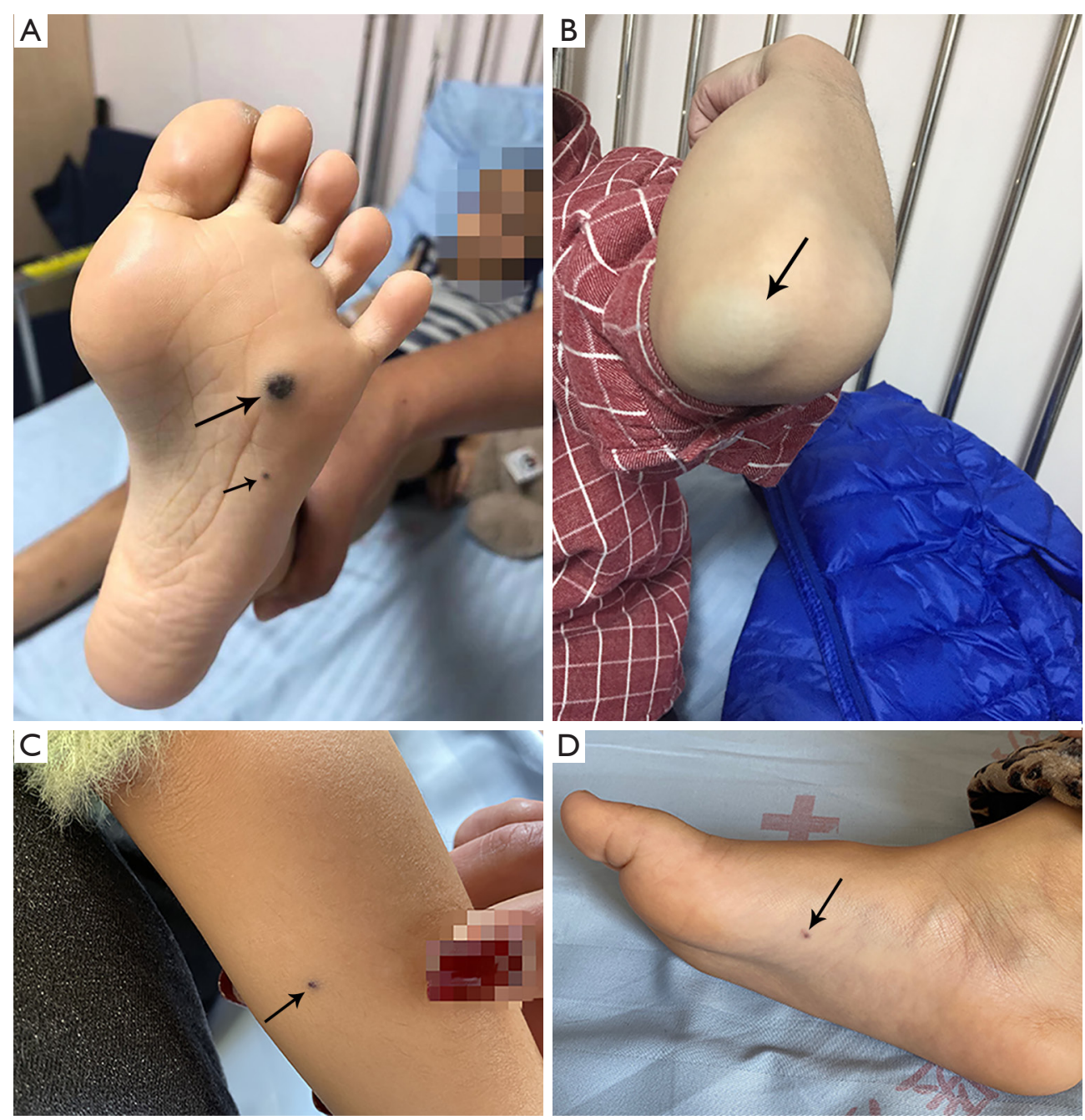

Figure 1 Physical examination identified cutaneous lesions on skin (marked by the black arrows). (A) Rubbery cutaneous venous malformations over left sole (patient \#1). This image is published with the patient's consent; (B) subcutaneous venous malformation over left elbow, size was $3.5 \mathrm{~cm} \times 3.0 \mathrm{~cm}$ (patient \#1); (C,D) blue vascular papules over left forearm and right sole (patient \#7).

and transferrin saturation were lower, total iron binding capacity were higher, which indicated IDA. Coagulation function tests showed normal except D-dimer, most cases showed increased D-dimer levels at baseline (except patient $6 \#$, the maximum values of D-dimer were $4.54,2.03,2.83$, $0.80,1.04,2.28,2.97 \mathrm{mg} / \mathrm{L}$ respectively), which declined after sirolimus treatment (ranges of patients \#1, \#2, \#5, \#7 were $1.10-3.43,0.40-1.62,0.18-0.55,0.45-0.80 \mathrm{mg} / \mathrm{L}$, respectively). Faecal occult blood was positive in all patients.

\section{Radiological examinations}

Ultrasound (US) and computed tomography (CT) scan of abdomen were performed in all patients, 2 patients had positive results, multiple strong echoes in liver, spleen with acoustic shadows (the maximum size was $5.5 \mathrm{~mm} \times 3.2 \mathrm{~mm}$ ) of patient \#1 were detected by abdominal US, which may be calcifications, intussusceptions were observed by US when he had three severe abdominal pains; several strong echoes with acoustic shadows in liver of patient \#4 were detected by abdominal US. Abdominal CT scan showed multiple spotted calcification in liver and scattered nodular low density shadow in bowel wall with multiple punctate calcifications (patient \#1, Figure 2A,2B). CT scan of neck and chest was performed in patient \#1, detected different sizes of soft tissue mass shadows in thyroid with calcification 

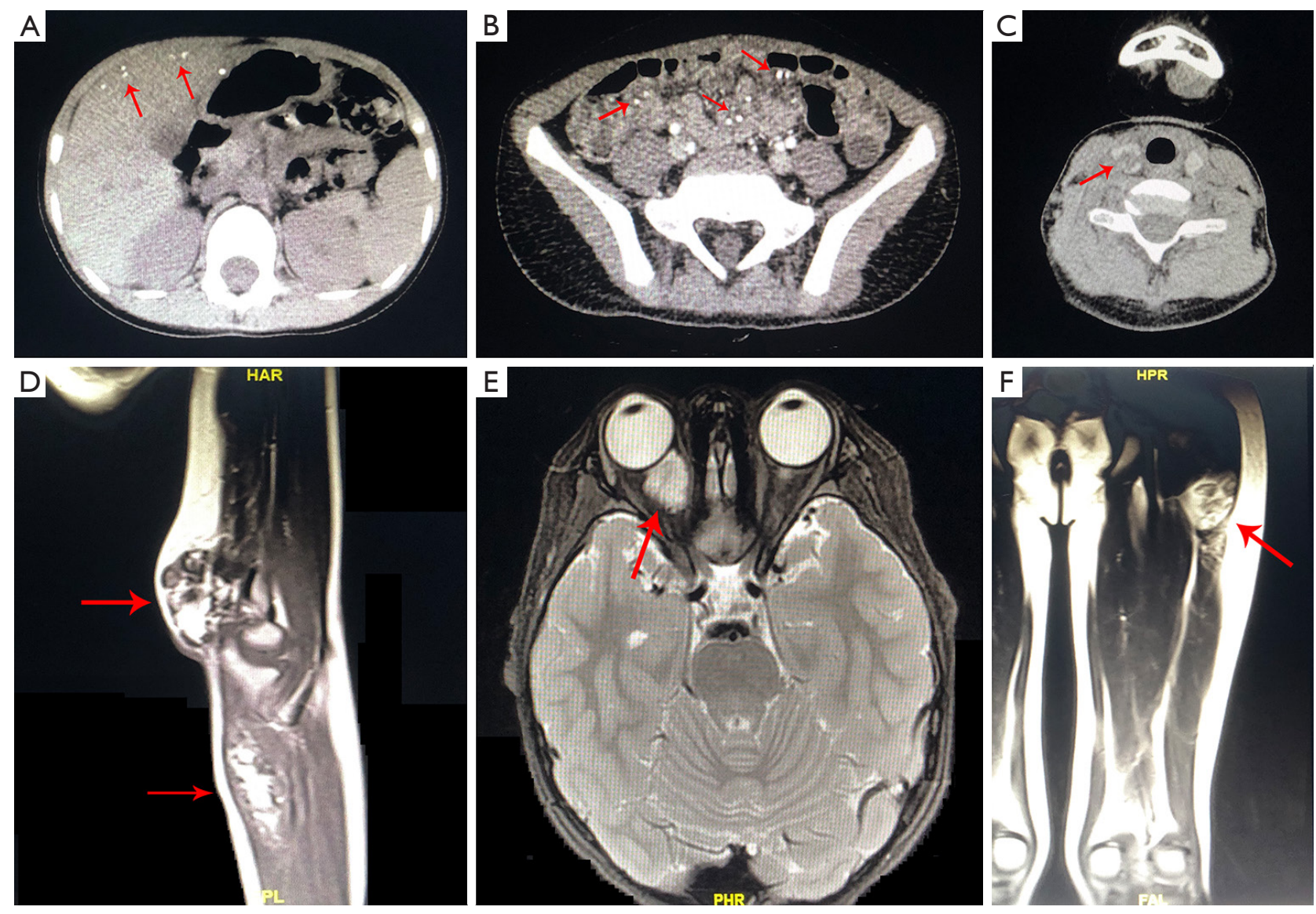

Figure 2 Computed tomography and magnetic resonance imaging suggested venous malformations (marked by the red arrows). Patient \#1: $(\mathrm{A}, \mathrm{B})$ abdominal CT showed multiple spotted calcification in liver and scattered nodular low density shadow in bowel wall with multiple punctate calcifications; (C) neck CT showed a soft tissue mass shadow in thyroid, size was $9 \mathrm{~mm} \times 8 \mathrm{~mm}$; (D) left elbow MRI showed multiple ball-like T1WI isointensity lesions involving the subcutaneous and intermuscular septum, the maximum diameter was $3 \mathrm{~cm}$; (E) ocular MRI showed a mass with clear boundary in muscle vertebra of the right retroocular space, which showed hyperintense in T2WI, size was $1.4 \mathrm{~cm} \times 1.6 \mathrm{~cm} \times 1.3 \mathrm{~cm}$. Patient \#2: (F) left thigh MRI detected longitudinal striped T2WI hyperintense in lesions involving the intermuscular septum, with punctate enhancement. CT, computed tomography; MRI, magnetic resonance imaging; T1WI, T1-weighted imaging; T2WI, T2-weighted imaging.

dots (Figure 2C), and multiple small nodules in both lungs. On left elbow magnetic resonance imaging (MRI) of patient \#1, multiple ball-like T1-weighted imaging (T1WI) isointensity and T2-weighted imaging (T2WI) hyperintense mixed with cord-like low signal lesions involving the subcutaneous and intermuscular septum were found, suggestive of low-flow VMs (Figure 2D), his ocular MRI revealed a mass with clear boundary in muscle vertebra of the right retroocular space, which showed hyperintense in T2WI with inhomogeneous enhancement (Figure 2E). MRI of left thigh detected longitudinal striped T1WI isointensity and T2WI hyperintense in lesions involving the intermuscular septum, with punctate enhancement (patient \#2, Figure 2F).

\section{Endoscopic examinations}

All patients underwent gastroscopy and ileo-colonoscopy, and 6 patients finished the whole GI screening with capsule endoscopy (CE) examinations, all underwent an average of 3.25 gastroscopy (range, 1-9), 2.38 ileo-colonoscopy (range, 1-6), and $1 \mathrm{CE}$ (range, 0-2) each. The locations of VMs were listed as follows: 4 cases in stomach; 1 case in duodenum, 7 cases in jejunum, 8 cases in ileum and 3 cases 

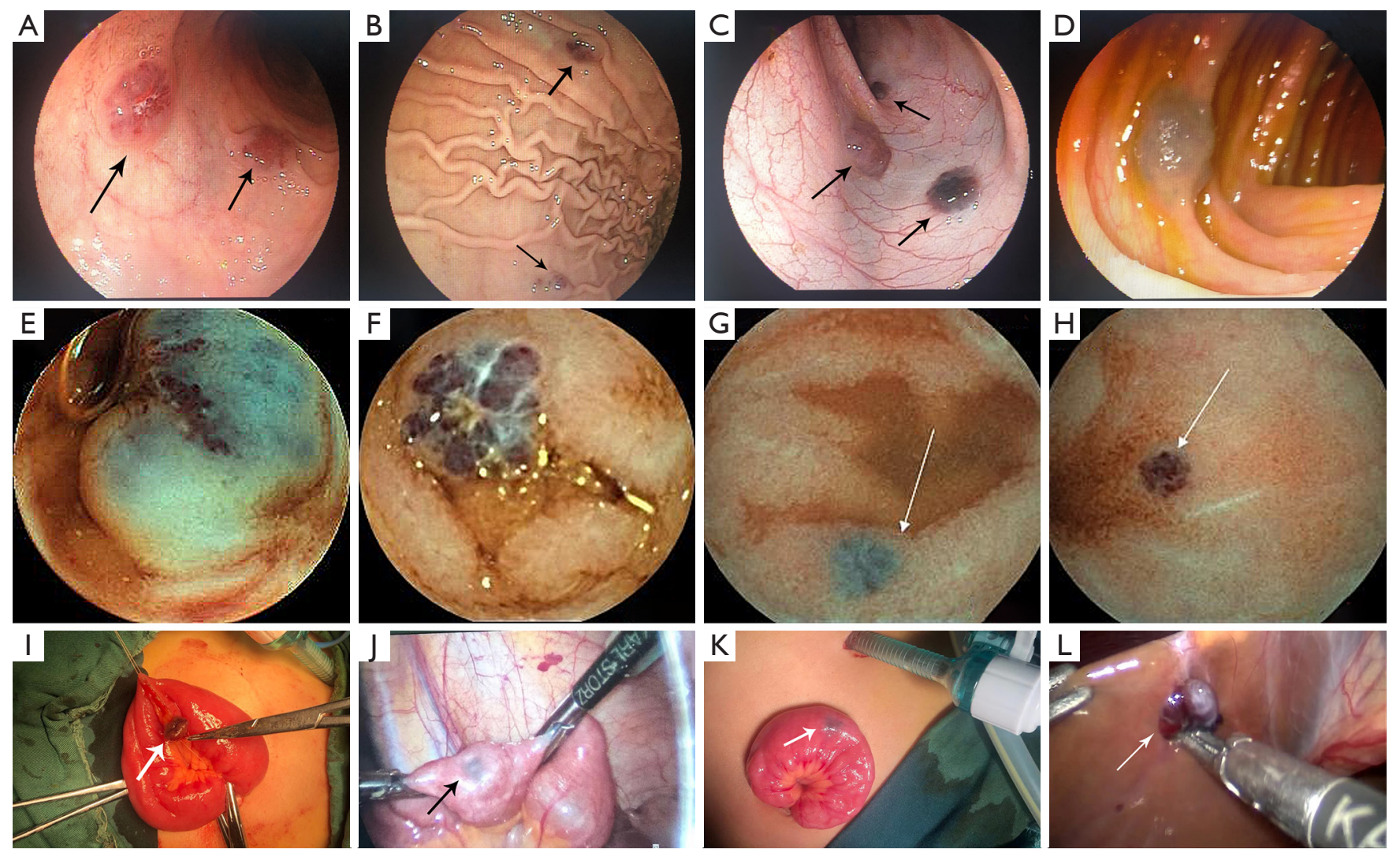

Figure 3 ndoscopy and operation showed multiple, bluish-purple, tender, nodular VMs in GI tract (marked by arrows). (A-C) Multifocal rubbery VMs in descendant duodenum, gastric body and ascending colon (patient \#1); (D) VMs in ascending colon $(1 \mathrm{~cm} \times 1 \mathrm{~cm}$, patient \#2); (E-H) CE showed various appearances of VMs in jejunum (patients \#2, \#3, \#5 and \#7, respectively); (I,J) surgery revealed two dark purple VMs on wall of terminal ileal and middle jejunum respectively (patient \#5); (K,L) surgery revealed two dark purple VMs on serosal of terminal ileal and beside of ligamentum teres hepatis respectively (patient \#7). VMs, venous malformations; CE, capsule endoscopy.

in colon. A great variation in the number of lesions were founded, form 2 to more than 30. CE provided a $100 \%$ positive diagnostic yield for BRBNS lesions. Generally, endoscopic examinations all showed multiple, bluishpurple, tender, nodular VMs in GI tract, sometimes showed submucosal protrusive lesion (Figure 3).

\section{Pathological examinations}

Postoperative VMs specimens of all patients were sent for pathological examinations, skin specimens of 2 patients were also included (patients \#1 and \#5). The surgery slices showed that there were proliferative and dilated cavernous vascular cavities under the mucosa lined by normal or thin or flat endothelium with varying amounts, the cavities were full of red blood cells, some of which could be seen thrombus organization and calcification in different degrees, suggesting VMs.

\section{Molecular diagnosis}

Four patients underwent genetic testing. There was no mutation associated with the disease in the whole exon sequencing of patients \#1 and \#5, and no somatic mutations in TEK (patients \#5, \#6 and \#7).

\section{Treatment and follow-up}

Supportive therapy included oral iron supplementation, drug hemostasis and blood transfusion in all (100\%), patient \#1 need for recurrent blood transfusions (on average more than one transfusion per month). Four patients received oral sirolimus capsule (patients \#1, \#2, \#5 and \#7). The initial dose is $1.0 \mathrm{mg} / \mathrm{m}^{2}$ of body surface area once a day 
or twice daily. The plasma concentration of sirolimus was measured at 2 weeks, 1, 3, 6 months, then every 6 months if the concentration is stable. Dosage was adjusted to maintain trough level between 5 and $15 \mathrm{ng} / \mathrm{mL}$, according to the condition of the patients. Only 3 patients (patients $\# 1$, \#2 and \#7) were underwent endoscopic sclerotherapy of lauromacrogol, number of treated lesions were varied from 3 to 15 (only diameter more than $1 \mathrm{~cm} \times 1 \mathrm{~cm}$ were treated with sclerotherapy). GI surgery was performed in all patients (100\%) and included: exploratory laparotomy and partial resection of intestinal VMs in 2, partial resection of intestinal VMs under laparoscope in 4, partial ileum resection under laparoscope in 1 , small intestinal resection and anastomosis under laparoscope in 1 (lesions concentrated in that segment), number of treated lesions were varied from 2 to 10 . In patient \#7, two dark purple VMs (size $0.5 \mathrm{~cm} \times 0.5 \mathrm{~cm}$ ) were removed at the edge of left lobe of liver and beside ligamentum teres hepatis during surgery, these lesions were not be seen under abdominal US and CT. Endoscopic therapies and surgery were invasive and technically demanding, could not eradicate all the lesions and prevent relapse. For instance, a transient rise in $\mathrm{Hb}$ was recorded after these aforementioned regimens, but it would fall again soon, and repeated those treatments are needed. Patients were followed for 1 months to 11 years, all patients insisted on iron supplementation, but still need to be combined with other treatments. Patient \#1 received sirolimus for more than 8 years with limited success, still experienced intermittent GI bleeding and severe anemia with $\mathrm{Hb}$ level of 36.2-120.0 g/L (Figure 4A), required regular blood transfusion (but frequency was lower than before), gastroenteroscopy was revisited every 3-4 years. For patient \#2, sirolimus increased his $\mathrm{Hb}$ level (120-132 g/L), eliminated blood transfusion, and reduced iron supplementation (Figure 4B). Patients \#5 and \#7 started sirolimus treatment within 1 month, therapeutic effect still needs long-term follow-up. GI bleeding were relieved of patients \#3, \#4, and \#6 after operation, Hb level could be maintained above $80 \mathrm{~g} / \mathrm{L}$. Unfortunately, patients \#3, \#4 and \#8 lost to follow-up after discharge due to personal reasons. In addition, we found patient \#1 had growth retardation (only slight decrease of growth hormone), the height and weight of 6 and 12 years old were $16.5 \mathrm{~kg} / 107.2 \mathrm{~cm}$ ( $<3 \mathrm{rd}$ percentile) and $25.8 \mathrm{~kg} / 136.7 \mathrm{~cm}$ ( $<3 \mathrm{rd}$ percentile), and some emotional problems appeared after 10 years old, included depression, inferiority and inattention, so he accepted the psychological intervention treatment for a year, other patients' height and weight were between 10th and 75th percentile.

\section{Discussion}

BRBNS can occur at any age and does not have a sex predilection, cutaneous manifestations typically present at birth or early childhood, the maximum age of diagnosis reported in literature was 89 years old (4), about $55 \%$ occur within 18 years old. The estimated incidence of BRBNS is very lows, about $1: 14,000$ births (5), it is usually a sporadic disorder. Recently, Soblet et al. identified somatic mutations in TEK, the gene encoding TIE2 (an endothelial cell tyrosine kinase receptor for angiopoietins), as the principal cause of BRBNS, which has also been implicated in spontaneous and familial VMs, and T1105N-T1106P is recurrent in this disease. In contrast to common unifocal VM, multifocal malformations are associated with two somatic activating mutations on the same allele (double cis mutations) (6). All our patients presented with no family history of BRBNS, we did not detect mutations in one tissue each from patients \#5, \#6 and \#7, likely due to below-detection levels of mutant cells in heterogeneous samples. As previously mentioned, genetic test helps to find the mutated genes and can distinguish from other VMs, is very important for clinical treatment decision, such as sirolimus. Distinct from other vascular malformations, localized intravascular coagulopathy resulting from chronic venous blood clotting is one of the characteristic manifestations of VMs (including BRBNS), an increasing number of studies link VMs to coagulation abnormalities, especially to elevated D-dimer and decreased fibrinogen (7). In our study, $87.5 \%$ of patients had elevated D-dimer levels of greater than $0.5 \mathrm{mg} / \mathrm{L}$, and the increase degree was positively correlated with the number and lesions' extent of VMs, demonstrated that elevated D-dimer was associated with large size, diffuse and multifocal VMs.

One of the most common findings of BRBNS is cutaneous lesions, typical lesions are blue or purple lesions with a rubbery feel, and the size of lesions vary from small papules to large disfiguring nodules, some patients complain of painful lesions (5\%) (8). Ninety-four-point-eight percent patients have cutaneous lesions according to the latest statistical analysis, extremities were most frequently involved $(70.0 \%)$, followed by trunk (66.4\%) and head $(30.8 \%)$, about $10.4 \%$ also had perineal involvement, the onset of lesions are usually at birth (49.4\%) (9), fetal period is rare, but it has also been reported. Monrigal et al. reported two prenatal cases of large cutaneous vascular malformations that were associated with BRBNS, one was a left cystic 
A

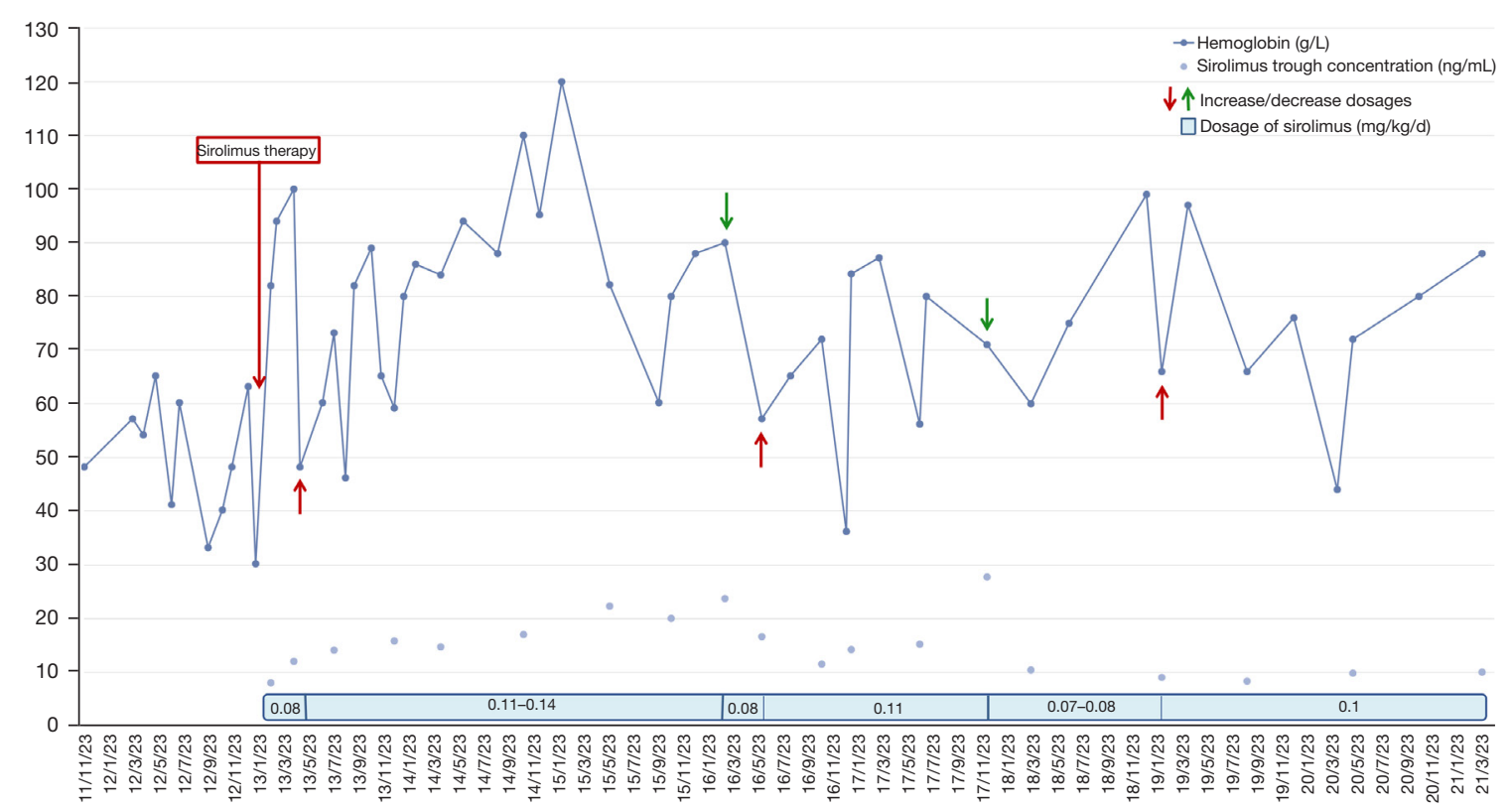

B

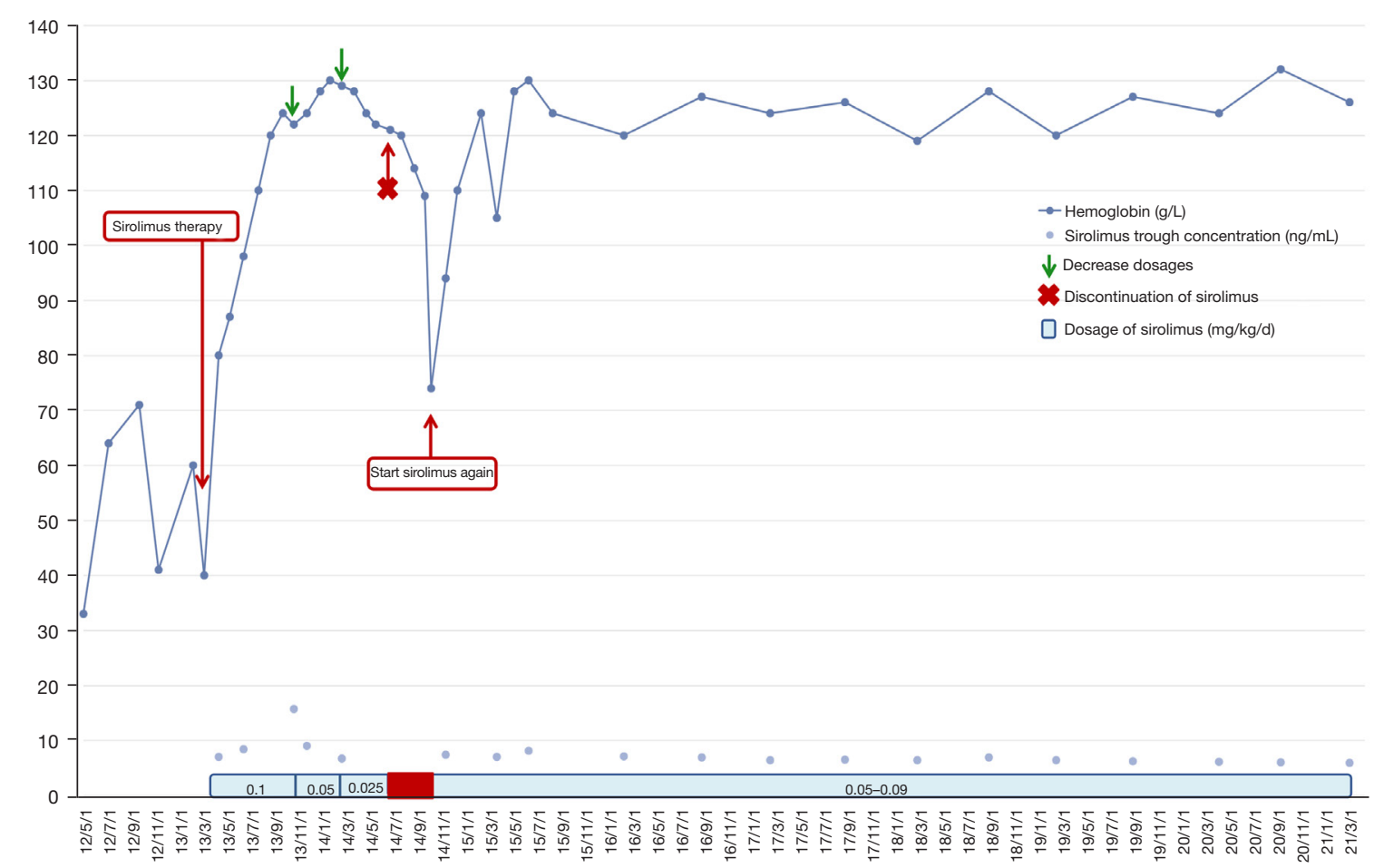

Figure $4 \mathrm{Hb}$ levels before and after sirolimus therapy. Patients had frequent drops in Hb level and almost received red blood cell transfusions before sirolimus therapy, after starting sirolimus. (A) Although $\mathrm{Hb}$ of patient \#1 dropped to the level that required blood transfusions, the frequency decreased from per month to half a year; (B) Hb levels of patient \#2 were normal and GI bleeding disappeared, when stopped using sirolimus after 1.25 years, soon a greater decrease in $\mathrm{Hb}$ level happened on the third month after discontinuation of sirolimus, once sirolimus is used again, Hb levels rose and remained above $120 \mathrm{~g} / \mathrm{L}$. GI, gastrointestinal; Hb, hemoglobin. 
axillary lesion at 29 weeks and another was an exophytic midline dorsal tumoral lesion at 25 weeks' gestation (10). Among our cases, $87.5 \%$ patients had cutaneous lesions, extremities were involved (100\%), trunk $(57.1 \%)$ and head (28.6\%), 5 patients started during infancy (two of them from birth). Skin lesions rarely cause debilitating disease, in contrast, the GI lesions are a major cause of morbidity, were found in $77.2 \%$ patients, may appear in any position from mouth to anus, the most common symptoms are GI bleeding (69.2\%) and secondary IDA, $65.8 \%$ began in childhood, manifested as dominant bleeding or occult blood in stool. In our cases, all GI lesions led to chronic bleeding and IDA. Besides, we have a case show growth retardation and emotional problems, similar case has been reported (11), the phenomenon indicated a maximum possible causal relationship between IDA and growth retardation. Previous literature has reported that chronic anemia can also have an impact on growth hormone and insulin-like growth factor-I (12), abnormal growth and mental retardation were also non-hematologic effects of IDA (13).

Patients were diagnosed with BRBNS based on clinical, radiological, and endoscopic findings, for many patients, the final diagnosis was established by endoscopic findings of lesions which attributed to severe IDA in their early childhood. Soblet $e t$ al. set forward the diagnostic criteria for BRBNS: (I) the presence of GI lesions; (II) the presence at birth of one "dominant" visible lesion ( $>10$ times the area of all other visible lesions), in combination with 10 or more small $(<2 \mathrm{~cm}$ diameter) cutaneous lesions; the latter to include hyperkeratotic, palmoplantar lesions (6). In addition, small intestine is the most common site of GI tract involvement, as a result, patients may present with secondary chronic IDA from recurrent GI hemorrhage, which may be difficult to detect. However, due to its low incidence and atypical clinical symptoms, it was easily misdiagnosed in early stage. In our cases, we present a 14-year-old boy (patient \#6) who had atypical skin lesions for 13.5 years and refractory IDA for 5 years without GI dominant bleeding, chest and abdomen CT, GI endoscopy showed no lesions causing anemia, all of the above results lead to clinical confusion and failure to consider BRBNS, a CE was then performed and revealed multiple vascular lesions that lead to this diagnosis, and treated his IDA under laparoscope and resection of GI lesions. This case demonstrates that IDA can be the only symptom of GI lesions in BRBNS. CE is a non-invasive, reliable imaging technique, well accepted and tolerated by patients, can evaluate the entire small bowel and identify the distribution of BRBNS lesions, also be used for the diagnosis of BRBNS. In our cases, 4 cases $(50 \%)$ were considered BRBNS owning to CE. In addition, this disease can involve all parts of body, such as patient \#1 have 7 organs involved. VMs in important parts can cause serious consequences, for instance intracranial hemorrhage or embolization is an important cause of death (14). Therefore, it is necessary to comprehensively and systematically evaluate the VMs involvement of various organs. Significantly, BRBNS should be differentiated from other congenital vascular malformation syndromes such as hereditary hemorrhagic telangiectasia (Osler-Weber-Rendu syndrome), diffuse neonatal hemangiomatosis, familial glomangiomatosis, Klippel-Trenaunay-Weber syndrome, Maffucci syndrome, and mucosal VM syndrome $(2,8)$.

Currently, there is no uniform effective treatment of BRBNS, curing them completely is difficult, the most important step is monitoring the evolution of GI lesions and preventing severe bleeding, but should create individualized treatment for patients depends upon the site and severity of the lesions. Skin lesions generally do not require special treatment, and laser photocoagulation, sclerotherapy, or surgical resection options are only considered in terms of aesthetics and function (15). Endoscopic treatment is less invasive and can completely preserve the length of the bowel, so it is suitable for small number of lesions and endoscopic accessible lesions. Surgery should be considered when endoscopic treatment is not available or failed, and serious complications such as volvulus, intussusception, and intestinal perforation occur. Previous studies suggest that surgical treatment and endoscopic treatment have a relatively high recurrence rate, so anti-angiogenesis drug therapy is very necessary. Notably, sirolimus (rapamycin) is a novel antiangiogenic agent, which acts by inhibiting the mTOR pathway to reduce the size of VMs and control GI bleeding. Since Yuksekkaya et al. first reported that sirolimus can be used in treatment of children with BRBNS in 2012 (16), a total of 28 pediatric patients with BRBNS treated with sirolimus have been described, and the longest treatment duration reported to date was 9 years $(17,18)$, according to the literature review of Weiss et al., an average sirolimus dosage of $0.05 \mathrm{mg} / \mathrm{kg} / \mathrm{d}$ (range, $0.02-0.1 \mathrm{mg} / \mathrm{kg} / \mathrm{d}$ ) or $1.42 \mathrm{mg} / \mathrm{m}^{2}$ (range, $0.6-2 \mathrm{mg} / \mathrm{m}^{2}$ ) was used with mean sirolimus trough concentration of $7.5 \mathrm{ng} / \mathrm{mL}$ (range, $1-15 \mathrm{ng} / \mathrm{mL}$ ) (17). Different target trough concentration have been recommended in the literature, originally, the recommended beginning 
dosage is $1.6 \mathrm{mg} / \mathrm{m}^{2} / \mathrm{d}$ divided into two doses given every 12 hours, with subsequent dosage adjusted to maintain a goal drug trough level of $10-15 \mathrm{ng} / \mathrm{mL}(19,20)$, but successful management of BRBNS has been described anecdotally at lower trough concentration of $1-5 \mathrm{ng} / \mathrm{mL}$ $(16,19,21,22)$. In our cases, 2 patients received sirolimus treatment more than 8 years, the dosage of sirolimus in patient \#1 was adjusted according to $\mathrm{Hb}$ fluctuation and clinical manifestations $(0.07-0.11 \mathrm{mg} / \mathrm{kg} / \mathrm{d})$, with mean trough concentration of $15.02 \mathrm{ng} / \mathrm{mL}$ (range, $8-27.72 \mathrm{ng} / \mathrm{mL}$ ), but clinical therapeutic efficacy is still not perfect. In patient \#2, range $0.025-0.1 \mathrm{mg} / \mathrm{kg} / \mathrm{d}$, with mean trough concentration of $7.4 \mathrm{ng} / \mathrm{mL}$ (range, $5.9-15.7 \mathrm{ng} / \mathrm{mL}$ ), but it is interesting to note that a greater decrease in $\mathrm{Hb}$ level, new skin lesions and melena happened on the third month after discontinuation of sirolimus, once sirolimus was used again, these symptoms improved rapidly. The trough concentration and therapeutic effect of our two patients are different at the same dosage, heterogeneity of patients is one reason, other reasons, such as mechanisms of action of sirolimus still need to be explored in depth. Sirolimus has potentially adverse effects on renal functions, bone marrow and cholesterol metabolism, regular checks of renal, liver, and hematological parameters were within normal ranges in our 2 cases. Thus, low-dose sirolimus may adequately reduce GI and intralesional bleeding, with minimal adverse effects, but it appears that chronic intestinal bleeding persisted in some of these cases, albeit at low levels (22).

\section{Conclusions}

The multifocal VMs of BRBNS commonly occur on skin and the mucous membrane of GI tract. Most patients develop severe IDA due to chronic GI hemorrhage and require lifelong iron supplementation and blood transfusion. $\mathrm{CE}$ is a non-invasive imaging technique, well accepted by children, is helpful to evaluate the cause of small intestinal bleeding and improve the diagnosis rate. Patients with short duration, mild symptoms and few VMs lesions can be treated by endoscopic therapies and surgery, but those treatments are invasive and technically demanding, could not eradicate all the lesions and prevent relapse. Sirolimus at a relatively low dosage effectively reduces the size of VMs, alleviates GI bleeding, abolishes transfusion dependence, and improves the quality of patient life with acceptable adverse effects. Further study is needed to explore the optimal dosage of sirolimus in the initial phase and maintenance stage. Long-term follow-up is important in BRBNS patients, for children, in addition to the impact of disease itself, we also need to pay attention to the impact of this chronic disease on physical and mental health.

\section{Acknowledgments}

The authors would like to thank all patients and families participating in the study.

Funding: None.

\section{Footnote}

Reporting Checklist: The authors have completed the AME Case Series reporting checklist. Available at https://dx.doi. org/10.21037/tp-21-238

Data Sharing Statement: Available at https://dx.doi. org/10.21037/tp-21-238

Conflicts of Interest: All authors have completed the ICMJE uniform disclosure form (available at https://dx.doi. org/10.21037/tp-21-238). The authors have no conflicts of interest to declare.

Ethical Statement: The authors are accountable for all aspects of the work in ensuring that questions related to the accuracy or integrity of any part of the work are appropriately investigated and resolved. The study was conducted in accordance with the Declaration of Helsinki (as revised in 2013). The study was approved by Ethical Committee of Children's Hospital of Fudan University (No.: 2015-130) and informed consent was taken from all patients' parent or legal guardian.

Open Access Statement: This is an Open Access article distributed in accordance with the Creative Commons Attribution-NonCommercial-NoDerivs 4.0 International License (CC BY-NC-ND 4.0), which permits the noncommercial replication and distribution of the article with the strict proviso that no changes or edits are made and the original work is properly cited (including links to both the formal publication through the relevant DOI and the license). See: https://creativecommons.org/licenses/by-nc-nd/4.0/.

\section{References}

1. Cho YM, Steen C, James J. Blue rubber bleb nevus 
syndrome: a rare cause of abdominal pain. ANZ J Surg 2020;90:619-21.

2. Baigrie D, Rice AS, An IC. Blue Rubber Bleb Nevus Syndrome. 2021 Jul 12. In: StatPearls [Internet]. Treasure Island (FL): StatPearls Publishing, 2021.

3. Isoldi S, Belsha D, Yeop I, et al. Diagnosis and management of children with Blue Rubber Bleb Nevus Syndrome: A multi-center case series. Dig Liver Dis 2019;51:1537-46.

4. Aron J, Couturier A, Sinayoko L, et al. An unusual cause of gastrointestinal bleeding in a hemodialysis patient. Hemodial Int 2018;22:E60-2.

5. Martinez CA, Rodrigues MR, Sato DT, et al. Blue rubber bleb nevus syndrome as a cause of lower digestive bleeding. Case Rep Surg 2014;2014:683684.

6. Soblet J, Kangas J, Nätynki M, et al. Blue Rubber Bleb Nevus (BRBN) Syndrome Is Caused by Somatic TEK (TIE2) Mutations. J Invest Dermatol 2017;137:207-16.

7. Aronniemi J, Långström S, Mattila KA, et al. Venous Malformations and Blood Coagulation in Children. Children (Basel) 2021;8:312.

8. Jin XL, Wang ZH, Xiao XB, et al. Blue rubber bleb nevus syndrome: a case report and literature review. World J Gastroenterol 2014;20:17254-9.

9. Pan Y, Li L, Li H. The clinical analysis of two hundred and fifty blue rubber bleb nevus syndrome cases. Sichuan Medical Journal 2019;40:1134-7.

10. Monrigal E, Gallot D, James I, et al. Venous malformation of the soft tissue associated with blue rubber bleb nevus syndrome: prenatal imaging and impact on postnatal management. Ultrasound Obstet Gynecol 2009;34:730-2.

11. Pan Y, Zhang L, Duan M, et al. Blue Rubber Bleb Nevus Syndrome: A Possible Cause for Growth Retardation and Pubertal Delay. Med Princ Pract 2019;28:294-6.

12. Soliman AT, De Sanctis V, Yassin M, et al. Growth and Growth hormone - Insulin Like Growth Factor -I

Cite this article as: $\mathrm{Xia} \mathrm{H}, \mathrm{Wu} \mathrm{J}, \mathrm{Huang} \mathrm{Y}$. Blue rubber bleb nevus syndrome: a single-center case series in 12 years. Transl Pediatr 2021;10(11):2960-2971. doi: 10.21037/tp-21-238
(GH-IGF-I) Axis in Chronic Anemias. Acta Biomed 2017;88:101-11.

13. Ghosh K. Non haematological effects of iron deficiency a perspective. Indian J Med Sci 2006;60:30-7.

14. Tomelleri G, Cappellari M, Di Matteo A, et al. Blue rubber bleb nevus syndrome with late onset of central nervous system symptomatic involvement. Neurol Sci 2010;31:501-4.

15. Hu Z, Lin X, Zhong J, et al. Blue rubber bleb nevus syndrome with the complication of intussusception: A case report and literature review. Medicine (Baltimore) 2020;99:e21199.

16. Yuksekkaya H, Ozbek O, Keser M, et al. Blue rubber bleb nevus syndrome: successful treatment with sirolimus. Pediatrics 2012;129:e1080-4.

17. Weiss D, Teichler A, Hoeger PH. Long-term sirolimus treatment in blue rubber bleb nevus syndrome: Case report and review of the literature. Pediatr Dermatol 2021;38:464-8.

18. Quan X, Xu CD, Liu P, et al. Clinical effect of sirolimus in treatment of blue rubber bleb nevus syndrome in children: a report of 2 cases and literature review. Zhongguo Dang Dai Er Ke Za Zhi 2020;22:1011-6.

19. Adams DM, Trenor CC 3rd, Hammill AM, et al. Efficacy and Safety of Sirolimus in the Treatment of Complicated Vascular Anomalies. Pediatrics 2016;137:e20153257.

20. Hammill AM, Wentzel M, Gupta A, et al. Sirolimus for the treatment of complicated vascular anomalies in children. Pediatr Blood Cancer 2011;57:1018-24.

21. Özgönenel B, Martin A. Low-dose sirolimus controls recurrent iron deficiency in a patient with blue rubber bleb nevus syndrome. Pediatr Blood Cancer 2015;62:2054-5.

22. Yokoyama M, Ozeki M, Nozawa A, et al. Low-dose sirolimus for a patient with blue rubber bleb nevus syndrome. Pediatr Int 2020;62:112-3. 